\title{
Functional Genomics Approach Using Mice
}

\author{
Young Hoon Sung \\ 'Samsung Biomedical Research Institute and Department of Molecular Cell Biology, \\ Sungkyunkwan University, School of Medicine, Suwon 440-746, Korea \\ Department of Food Biotechnology, Sungkyunkwan University, Suwon 440-746, Korea
}

Received 11 December 2003

\begin{abstract}
The rapid development and characterization of the mouse genome sequence, coupled with comparative sequence analysis of human, has been paralleled by a reinforced enthusiasm for mouse functional genomics. The way to uncover the in vivo function of genes is to analyze the phenotypes of the mutant animals. From this standpoint, the mouse is a suitable and valuable model organism in the studies of functional genomics. Therefore, there have been enormous efforts to enrich the list of the mutant mice. Such a trend emphasizes the random mutagenesis, including ENU mutagenesis and gene-trap mutagenesis, to obtain a large stock of mutant mice. However, since various mutant alleles are needed to precisely characterize the role of a gene in vivo, mutations should be designed. The simplicity and utility of transgenic technology can satisfy this demand. The combination of RNA interference with transgenic technology will provide more opportunities for researchers. Nevertheless, gene targeting can solely define the in vivo function of a gene without a doubt. Thus, transgenesis and gene targeting will be the major strategies in the field of functional genomics.
\end{abstract}

Keywords: Functional genomics, Gene targeting, Mouse, Mutagenesis, Transgenic

\section{Introduction}

The mouse has a long history as an invaluable model organism in the field of biological studies. It exhibits a short life span with the large litter size that is suitable for genetic studies. Since the mouse is very similar to humans in both genetical and physiological aspects, the mouse models closely resembling human diseases are usually adopted for studying

*To whom correspondence should be addressed.

Tel: 82-31-299-6135; Fax: 82-31-299-6435

E-mail: hwl@skku.ac.kr human pathogenesis. Moreover, one of the most efficient ways to reveal the function of a gene is to analyze the phenotypes of an individual mutant mouse in vivo. Since mice can be genetically engineered with relative ease, the number of studies using mutant mice has been dramatically rising with the increase of the number of mutated genes in the mouse, most of which were accomplished through the pronuclei injection or the homologous recombination in embryonic stem (ES) cells (Muller, 1999). The introduction of gene targeting strategy has revolutionized the field of biological science itself as well as that of mouse genetics.

As the importance of enriching the pool of mutant mice is being recognized among scientific communities with the completion of both human and mouse genome projects, the demands for developing efficient strategies of mutagenesis have significantly increased. Therefore, current interest is focused on random mutagenesis strategies, such as ENUmediated and gene-trap mutagenesis. Reasonably, these mutant mice are beneficial to the identification of the genes that are involved in interesting traits and/or to the initial evaluation of the significance of a gene in both the genetical and physiological context. However, the planned or designed mutations are essential for studying both the physiological and molecular functions of a gene precisely. These objectives are just brought about through the generation of both targeted and transgenic mice since the potential of random mutagenesis is limited in the generation of allelic mutations. Based on it, we describe here the basic technologies that are used in the studies of mouse functional genomics and empathize the advantages of gene targeting and transgenesis.

\section{Gene Targeting}

Gene targeting, i.e., knockout or $\mathrm{KO}$, has enabled us to engineer the mouse genome at will. Pluripotency of the mouse ES cells and homologous recombination are the core principles for the introduction of specific mutations into the mouse genome. The pluripotent mouse ES cells are derived 


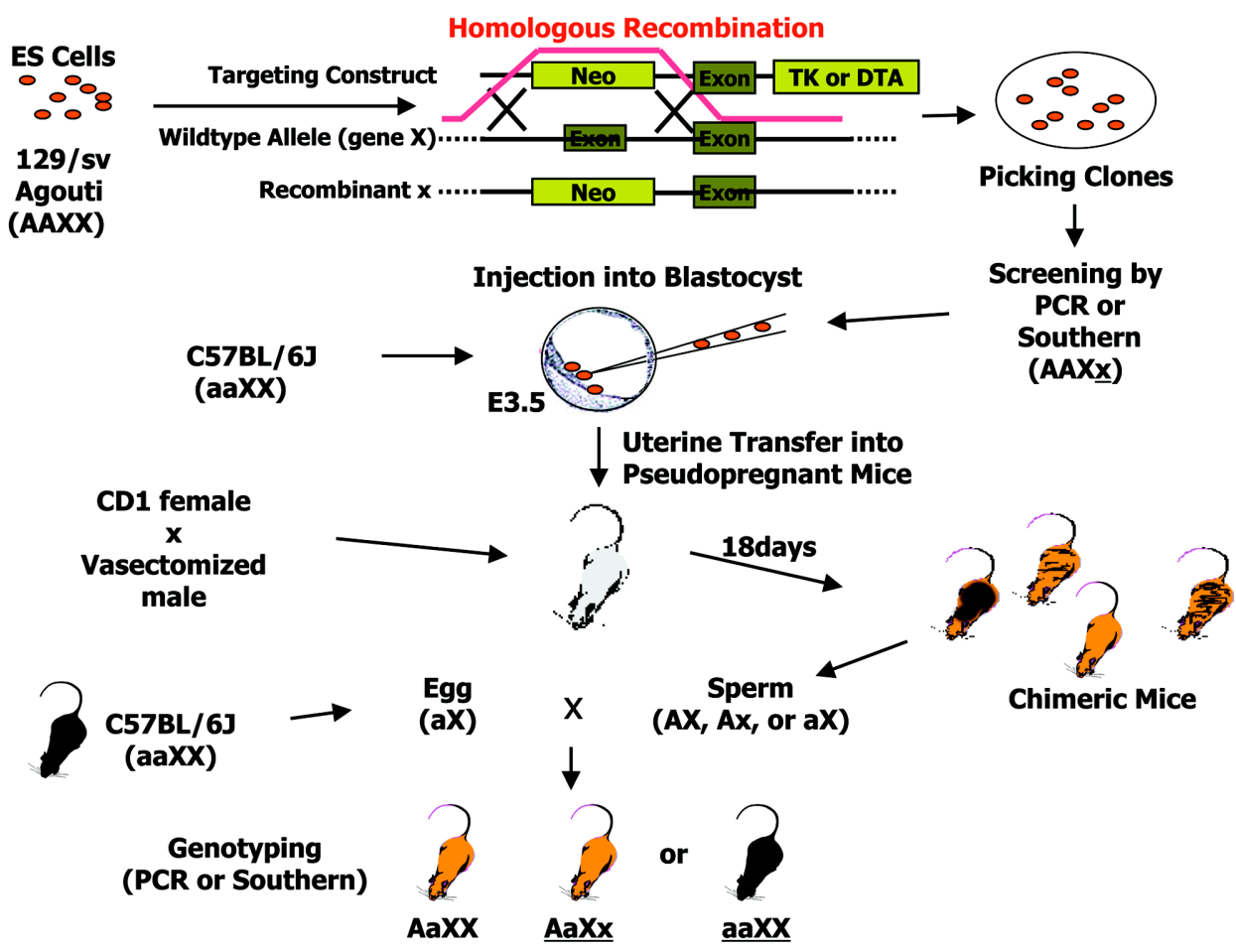

Fig. 1. Basic genetics in the production of knockout mice. ES cells are electroporated with a targeting vector. After selection, the clones are picked and screened by PCR or Southern blot analysis. The recombinant ES clones are injected into blastocysts that are then transferred into pseudopregnant mice to generate chimeric mice. Chimeras are crossed to C57BL/6J to obtain germ-line transmission. X, target gene; A, Agouti gene; Neo, neomycin phosphotransferase gene as a positive selection marker; TK or DTA, thymidine kinase or Diphtheria Toxin A, respectively, as negative selection markers.

from the inner cell mass of a mouse blastocyst and established in culture (Evans and Kaufman, 1981). These ES cells can reconstitute the germ-lines as well as all other tissues, thus a whole mouse, when re-injected into or aggregated with mouse embryos (Evans and Kaufman, 1981; Nagy et al., 1993). Even though they are being cultured for a long period of time in vitro, they can subsequently repopulate the germ-line in chimeric mice (Robertson et al., 1986).

Classical gene targeting The first gene-targeted mouse was generated using hypoxanthine phosphoribosyl transferase (HPRT) as a target via the homologous recombination in ES cells (Doetschman et al., 1987; Thomas and Capecchi, 1987). In order to minimize frequent random integrations, and thus increase the efficiency of gene targeting, a sophisticated selection method, known as "positive and negative selection", has been introduced (Mansour et al., 1988).

Describing the genetics underlying the gene targeting technology, Fig. 1 will be very useful for scientists who wish to understand the basics. Generally, ES cells are derived from mouse strain 129/sv that carries two wild-type alleles of the Agouti gene (AA), which is autosomal dominant, thus showing the Agouti color (similar to brown color) on their coat. The targeting vector for gene $\mathrm{X}$ that the scientist wants to knockout is constructed to contain two homologous regions flanking the positive selection marker (usually Neomycin phosphotransferase gene). The negative selection marker, such as thymidine kinase (TK) or Diphtheria toxin A (DTA) gene, is added to increase the yield of the proper homologous recombination. When this targeting vector is introduced into wild-type ES cells by electroporation, the homologous recombination will occur. Before the homologous recombinations take place, these wild-type ES cells have a genotype of AAXX. After the selection, the cloned ES cells are screened to find the homologous recombination event by Southern blot analysis or Polymerase Chain Reaction (PCR).

Afterward, the ES cell clone, heterozygous for gene $\mathrm{X}$ (AAXx), will be expanded and injected into wild-type blastocysts that are isolated from the cross between the C57BL/6 strain mice. Since these C57BL/6 mice have no wild-type Agouti gene (therefore they have a black coat color) and the target gene $\mathrm{X}$ is still intact, their genotype is aaXX. By microinjection, these ES cells (AAXx) are fused to the inner cell mass of blastocysts (aaXX), which are then transferred to the uterus of the pseudopregnant foster mother, resulting in the production of chimeric mice with the genotypes of both AAXx and aaXX.

These chimeric mice will have genotypes of $\mathrm{AX}, \mathrm{Ax}$, or aX in their germ cells (AX and Ax from injected ES cells, and aX from host blastocyst). If these are mated with wild-type C57BL/6 mice (only aX in their germ cells), the genotypes of the newborns will be AaXX, AaXx, or aaXX. What we want 
among these three is heterozygous knockout, Xx. Therefore, if one can see only black mice from these newborns, they must be aaXX that is not gene-targeted and if one gets Agouti color mice, one half of them $(\mathrm{AaXx})$ will be the properly targeted heterozygous knockout mice.

Conditional gene targeting Though the classical gene targeting strategy can successfully introduce specific mutations into the mouse genome, there are several disadvantages in employing this approach. First, since it uses the germ-line mutation, mutations in developmentally essential genes will induce embryonic lethality, which makes it impossible to study the gene function in adult stages. Secondly, it is limited for studies of the gene functions in the context of the specificity for both the cell lineage and the developmental progression. Finally, it is impossible to establish the models that closely resemble the somatically acquired human diseases. These limitations urged scientists to develop new targeting strategies. The site-specific recombination systems, such as Cre/LoxP from bacteriophage $\mathrm{P} 1$ and Flp/Frt systems from yeast, have enabled scientists to achieve the tissue-specific gene targeting. At first, these systems were successful in expressing a transgene in transgenic mice or ES cells; its translation is blocked by the presence of the artificial stop codon, which is designed to be deleted by Cre/Flp recombinase (Orban et al., 1992; Dymecki, 1996). This success led the T cell-specific DNA polymerase $\beta$ gene targeting (Cre-LoxP system) and the pituitary glandspecific Retinoblastoma gene targeting (FLP/FRT system) (Gu et al., 1994; Vooijs et al., 1998). Since the application range of the conditional gene targeting system is limited by the regulation of these recombination systems, diverse strategies have been developed to spatiotemporally regulate the Cre recombinase expression in vivo (Lewandoski, 2001).

Knock-in We have explained the strategies that can completely make null mutations. However, the subtle modification of these, so-called "knockin", has allowed the replacement of the endogenous gene. By fusing a cDNA to the coding sequence in frame, we can replace one gene with another, thereby comparing the role of the gene with the others. One of the examples is the knockin study showing the functional redundancy between cyclin D1 with cyclin $\mathrm{E}$ in mice (Geng et al., 1999). Since these two proteins are the major cyclins expressed in G1, they are referred to as G1 cyclins. These knockin mice express cyclin $\mathrm{E}$ with exactly the same pattern of cyclin D1 expression in vivo. This strategy rescued all phenotypic manifestations of cyclin D1 deficiency, thereby showing the functional redundancy of G1 cyclins.

Through knockin strategies, we can introduce a subtle mutation, such as deletions, substitutions of domains or even a point mutation, into the endogenous gene. For example, NF$\mathrm{\kappa B}$, a well-characterized transcription factor, strictly regulates both the innate and adaptive immune responses as well as inflammation. It forms a complex with $\mathrm{I} \kappa \mathrm{B}$, which negatively regulates its function. NF- $\mathrm{KB}$ is activated by dissociating from $\mathrm{I} \kappa \mathrm{B}$, which is phosphorylated by IкB kinase (IKK), composed of IKK $\alpha$, IKK $\beta$ and IKK $\gamma / \mathrm{NEMO}$, and then degraded. By replacing the wild-type allele with $\mathrm{IKK} \alpha^{\mathrm{AA}}$, an activationdefective mutant allele, the specific role of IKK $\alpha$ in the control of mammary epithelial proliferation was uncovered (Cao et al., 2001).

With the help of these gene-targeting strategies, we can definitively achieve the elimination or the replacement of genes of interest composing the mouse genome. Moreover, these technologies enable us to engineer the chromosome itself (Yu and Bradley, 2001), which is impossible to accomplish with any other genetic strategy. Further development of sophisticated technologies regulating the homologous recombination will eventually enable scientists to target a gene of interest in any stage of mouse development and in any cell lineage and tissue or organ at a desired time point.

\section{Transgenic Mice}

The establishment of transgenic technology has opened a new era in the field of biological science. Transgenic studies have attacked the gene functions in waves. Generally, transgenic mice are generated by microinjecting the DNA fragments into the pronuclei of fertilized eggs. This DNA fragment contains new genetic information and is integrated into the genomic DNA, which is transmitted to the next generation. The first transgenic mice were produced with the transgene, composed of mouse metallothionein-I gene promoter and the structural gene of rat growth hormone (Palmiter et al., 1982). This transgene confers on mice with a new potential to manifest gigantism when fed with extra zinc, thereby showing the functional transmission of new genetic information.

Specific targeting of the transgene expression Transgenic mice allowed the researchers to monitor the transgene expression in all cell lineages, at any desired stage of development and in postnatal animals, thereby providing new tools to investigate the cis-elements and promoters that are involved in transcriptional regulation. For example, in transgenic mice expressing the human growth hormone (hGH) under the regulation of the elastase I promoter, the hGH expression was directed to the pancreatic acinar cells, which exactly corresponds to that of the endogenous elastase I gene (Ornitz et al., 1985). Later, it was also demonstrated using transgenic mice that the enhancer of this elastase I regulatory region was responsible for both the expression in specific cell-lineage and the developmental onset of the expression (Hammer et al., 1987).

Concomitant with these studies, the availability of the wellcharacterized promoters allows the targeting of the transgene expression in specific cell lineages or tissues and organs. For example, several telomerase reverse transcriptase subunit 
(TERT)-overexpressing transgenic mice are generated. The chicken $\beta$-actin promoter and CMV early enhancer element permitted the TERT transgene expression in a variety of tissues (Artandi et al., 2002). The 5'-regulatory region of the bovine keratin $\mathrm{K} 5$ gene targeted the mTERT expression to basal keratinocytes (Gonzalez-Suarez et al., 2001). The cardiac-specific mouse $\alpha$-myosin heavy chain (MHC) promoter was employed to direct the expression of the wildtype human TERT to cardiac muscle cells (Oh et al., 2001). In order to express TERT transgene in hematopoietic stem cells (HSCs), the major histocompatability class I gene $\mathrm{H}-2 \mathrm{~K}^{\mathrm{b}}$ promoter was used (Allsopp et al., 2003).

Besides adopting tissue-specific promoters, the transgenic expression can be modulated by employing several inducible strategies, such as the tetracycline-inducible (Efrat et al., 1995) and site-specific recombination systems (i.e., Cre/LoxP or Flp/Frt system (Orban et al., 1992; Dymecki, 1996)). Technical progress will enable us to examine the in vivo role of a gene in specific tissues at a desired time point.

Transgenic mouse in studying pathophysiology Diseaserelated phenotypes could be reconstituted in mice through the expression of the pathogenic mutant alleles of a gene. Mutations in specific glycine residues of the pro-alpha 1 (I) collagen gene are associated with the inherited human disease, osteogenesis imperfecta type II. In transgenic mice expressing the mutant pro-alpha 1 (I) collagen gene with substitutions in a glycine residue, a dominant lethal phenotype, which is a characteristic of this human disease, was observed (Stacey et al., 1988).

The transgenic approach is extensively used both to establish heritable tumors in vivo and to reveal the oncogenic potential of a gene. Among them, the Metallothionein I promoter-driven SV40 T Ag expression induces heritable brain tumors in transgenic mice (Brinster et al., 1984). Another heritable tumor can also be induced in the pancreatic $\beta$ cells of the transgenic mice expressing SV40 T Ag under the regulation of the rat insulin II promoter (Hanahan, 1985). The c-myc oncogene driven by immunoglobulin enhancers leads to lymphoid malignancies in transgenic mice within a few months of their birth (Adams et al., 1985).

In addition to the specific expression of a pathogenic transgene, transgenic technology is utilized to examine the biological functions of the specific cell lineage by eliminating them in vivo. This is accomplished by targeting the expression of a toxic gene, such as Diphtheria toxin, in specific cell lineages. The Elastase I promoter is known to restrict the transgene expression in pancreatic acinar cells in mice (Ornitz et al., 1985; Hammer et al., 1987). The transgenic expression of the Diphtheria toxin (DT) A gene under the control of the elastase I promoter/enhancer specifically eliminates pancreatic acinar cells, thereby resulting in mice that lack a normal pancreas (Palmiter et al., 1987). This strategy was further developed to ablate specific cell-lineages at certain time point (spatiotemporal cell ablation). While the membrane-anchored form of the heparin binding EGF-like growth factor (HB-EGF precursor) acts as a DT receptor in humans, mouse orthologue does not. Therefore, when the human DT receptor is expressed in liver using the albumin promoter, DT specifically kills the hepatocytes only in these transgenic mice (Saito et $a l ., 2001)$. This strategy could be applied to eliminate certain types of cell-lineages at a desired time point.

\section{Disruption of endogenous gene function: Dominant negative mutations and RNA interference Dominant-} negative mutations are usually carried out for the elimination of the activities of the wild-type gene products. The expression of dominant-negative mutant genes will compete with and eventually disrupt the endogenous gene functions. This method is specially useful when the in vivo functions of a gene cannot be observed through classical gene targeting for it causes several obstacles such as early embryonic lethality homeostasis and functional redundancy. For example, ASC-2, a recently isolated transcriptional co-activator, when eliminated through gene targeting, induced embryonic lethality, which prevented scientists from analyzing the role of ASC-2 in the postnatal stages (Kuang et al., 2002; Antonson et al., 2003). On the other hand, the transgenic mouse overexpressing the dominant-negative mutant of the ASC-2 gene displayed multiple mutant phenotypes in various organs, including eye defects (Kim et al., 2002). In this case, the genetic leakiness of the dominant-negative approach was rather beneficial to avoiding the embryonic lethality.

Another strategy for the disruption of the gene functions by silencing the endogenous gene expression is RNA interference, which will be described in a later section.

Transgenic insertion as a mutagen Besides transmitting new genetic information to the mouse germ-line, the transgenic insertion itself can be used as a mutagenic tool. Transgenic insertions occur at random with insertional mutation frequencies of 5\% to $10 \%$ (Stanford et al., 2001). The mutated genes can be isolated using the inserted transgene as a probe. Using this approach, the Klotho gene, which is involved in the suppression of several ageing phenotypes, was identified (Kuro-o et al., 1997). In generating the transgenic mice overexpressing the rabbit type-I sodiumproton exchanger, researchers obtained several lines of transgenic mice that did not express this transgene. Among these mice, they identified the Klotho mutant mice. Recently, we also identified the mouse line that carries an insertional mutation (Choi et al., 2003). Using the chicken $\beta$-actin promoter and cytomegalovirus (CMV) early enhancer, we generated 7 lines of transgenic mice overexpressing mouse TERT. Among them, the homozygous offspring from one line showed severe ataxic phenotype. Positional cloning revealed that this transgenic insertion occurred in the intron 1 of the Unc $5 \mathrm{~h} 3$ gene, which resulted in transcriptional inactivation of this gene. Though this approach is laborious and not applicable for high-throughput screen, the same principle is 
successfully applied to gene-trap mutagenesis (described below).

RNA interference in mice Even with its short history, RNA interference (RNAi) using small interfering RNAs (siRNAs) has become a useful tool for studying gene functions in the mammalian system. RNAi using double-stranded RNA (dsRNA) has been generally applied to induce the sequencespecific gene silencing in the nematode worm Caenorhabditis elegans (Fire et al., 1998). Notably, the short dsRNA (small interfereing RNA, siRNA) is also effective in mammalian cells (Caplen et al., 2001; Elbashir et al., 2001; Hannon, 2002).

Stable expression of siRNA in vivo Currently, plasmidbased systems using the H1 or U6 promoter, driven by RNA polymerase III (RNA pol III), have been established to express short hairpin RNA (shRNA) molecules in mammalian cells (Brummelkamp et al., 2002; Paddison et al., 2002). With the selectable marker, this system is successfully applied for the stable silencing, i.e., knockdown, of the RasGAP gene expression in ES cells (Kunath et al., 2003). When these RasGAP-silenced ES cells were allowed to develop in the uterus, the embryos exhibited similar phenotypes that were observed in knockout embryos of this gene. Since the delivery of these siRNA-expressing plasmids relies on transfection, the retroviral vector system is useful for the introduction of siRNA-expression units into transfection-resistant cells such as primary cells (Barton and Medzhitov, 2002; Devroe and Silver, 2002). These retroviral systems, however, have several disadvantages, including the non-infection of non-cycling/ post-mitotic cells and the silencing of the shRNA expression during development from infected ES cells or embryos. These problems were overcome by utilizing the lentiviral vector system (Rubinson et al., 2003; Tiscornia et al., 2003). In addition to the ES cell- and lentiviral-mediated expression of siRNA, conventional transgenic techniques are available to generate siRNA-expressing transgenic mice (Hasuwa et al., 2002).

Regulation of siRNA expression in vivo Since RNA pol III promoters, such as the $\mathrm{U} 6$ and $\mathrm{H} 1$ promoters, are active in all tissues, the use of the RNA polymerase II (RNA pol II)dependent promoter is essential for the generation of tissuespecific knockdown mice. By employing both a Ribozyme fusion to cut off the 5' 7-methylguanosine $\left(\mathrm{m}^{7} \mathrm{G}\right)$ cap structure and a MAZ site for RNA pol II pausing, an effective in vivo application of siRNA can be achieved using the RNA pol IIdependent promoters (Shinagawa and Ishii, 2003). In this study, researchers expressed the long dsRNA using the cytomegalovirus (CMV) promoter. This long dsRNA, transcribed by RNA pol II, are processed to possess the 5' $\mathrm{m}^{7} \mathrm{G}$ cap structures, which is essential for nuclear export. A MAZ site at the 3' end pauses the transcription by RNA pol II. Though the transport of long dsRNA to the cytoplasm induces the interferon (IFN) response, the removal of the $5^{\prime} \mathrm{m}^{7} \mathrm{G}$ cap structure by the fused ribozyme inhibits the export of dsRNA. In nucleus, these long dsRNA are processed to generate siRNA, which is transported into cytoplasm to exert its negative effect on the gene expression. By pronuclei injection of this construct, the researchers observed the knockdown phenotype of the Ski gene in mouse embryos.

The conditional knockdown strategies are designed for the temporal gene silencing as well. If the targeted gene is indispensable, the conditional regulation of siRNA rules out the potential lethality or growth abnormality. Recently, two groups reported the establishment of inducible knockdown systems adopting tetracycline-inducible U6 promoters (Matsukura et al., 2003; Wiznerowicz and Trono, 2003). Based on the transgenic approach, these strategies will be used to analyze the gene functions in vivo.

In summary, RNAi has been successfully applied to mammalian cells to achieve stable gene silencing in vitro. More recently, in combination with the transgenic technology, RNAi is being revealed as a new tool for studying in vivo functions in mice. Furthermore, the introduction of both the conditional and tissue-specific promoters will provide opportunities to regulate the RNAi spatiotemporally, broadening our scope of functional genomics studies in mice.

\section{ENU Mutagenesis in Mice}

Among various chemical mutagens, N-ethyl-N-nitrosourea (ENU) is the most powerful and widely used one. ENU usually induces point mutations by ethylating $\mathrm{O}$ or $\mathrm{N}$ in DNA and predominantly modifying $\mathrm{A} / \mathrm{T}$ base pairs. A series of mutations can yield a vast array of allelic changes, which affect gene function and structure, and thus result in obtaining null and partial loss-, as well as gain-, of-function alleles.

The major screening strategy of ENU mutagenesis is phenotype-driven. If there is an assay system for a specific phenotype, mutations can be identified. A number of screening methods have been used to find phenotypically interesting mutant mice. Those include both the genome-wide screening strategies to find the dominant, or recessive mutations and regional screening strategies to find recessive phenotypes. The latter employs chromosomal deletions or balancer chromosomes for identifying the affected gene (Fig. 2.; Balling, 2001).

Phenotype-driven screening The male mouse is treated with an appropriate dose of ENU followed by mating with a wild-type female, which will give birth to mutant offspring. Thereby, without a prediction, we can isolate the mutant gene that is involved in some phenomena such as development and pathogenesis. Although genome-wide screening strategies are usually successful for dominant phenotypes (Fig. 2A), the recessive mode of inheritance, which takes a major portion in human genetic disease, cannot be observed via this method. It 


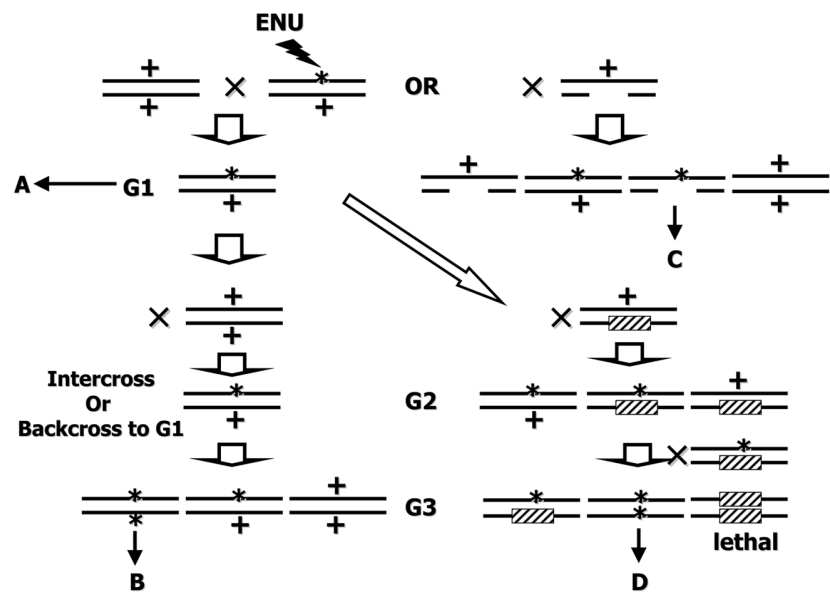

Fig. 2. Schematic explanation of the screening strategies used in ENU mutagenesis. (A) Genome-wide, dominant screen. The ENU-treated male is simply crossed with the wild-type female followed by the phenotypic assay of G1 mice. (B) Genome-wide, recessive screen. First, all G1 mice must be bred to produce G2 mice. Intercross between G2 mice or backcross to G1 mice is required to produce a homozygous mutant, G3, which will give rise to recessive phenotypes. (C) Regional screen for recessive phenotypes using chromosomal deletion. ENU-treated male mice must be mated with female mice, which have marked chromosomal deletions. If the mutations exist in the deleted region, affected G1 progenies will be hemizygous. Therefore, recessive phenotypes can be displayed by one-generation screen. (D) Regional screen for recessive phenotypes using balancer chromosomes. Since the dominant visible marker indicates the presence of wild-type allele and the homozygous mice are lethal, recessive phenotypes will be visualized in G3. +, wild-type allele; *, mutant allele; hatched box, chromosomal inversion.

takes at least 3 rounds of breeding to isolate the recessive mutations (Fig. 2B). As this genome-wide recessive screen is very laborious and time-consuming, regional screening strategies have been adopted. Two types of marker chromosomes are used for the regional screens: chromosomal deletions and balancer chromosomes (Rinchik, 2000).

If the chromosomal deletions are used, recessive phenotypes can be observed in hemizygous G1 progenies (Fig. 2C). It means that the affected gene must reside in the deleted region, which facilitate the cloning for the gene that is affected by ENU. Compared with chromosomal deletions, balancer chromosomes have some additional advantages (Fig. 2D). A large chromosomal deletion is frequently fatal to the well-being of mice. Furthermore, some genes show haploinsufficiency. The introduction of marked balancer inversions can solve these problems. Since the balancer chromosomes have a dominant visible marker and the homozygous mice are lethal, recessive phenotypes can be identified in G3 progenies. It also suppresses recombination events, which is enormously advantageous for the maintenance of mutant stocks.
The generation of chromosomal deletions and balancer chromosomes Since available chromosomal deletions and balancer chromosomes are limited, two other mutagenic strategies are employed to expand the list of these "genetic reagents". First, X-irradiation induces chromosomal deletions in ES cells (Thomas et al., 1998). Since these types of chromosomal deletions are not planned specifically, the exact deletion site must be determined afterwards. Second, defined chromosomal deletions or inversions can be introduced into the mouse genome using the site-specific homologous recombination in ES cells (Yu and Bradley, 2001). For instance, the chromosomal inversion, introduced between Trp53 (also known as p53) and Wnt3 on mouse chromosome 11 , is recessive lethal and dominantly marked with a K14Agouti transgene (Zheng et al., 1999). Recently, a regional recessive screen was successfully conducted using this balancer chromosome (Kile et al., 2003).

Difficulties in identifying mutated genes Since the major screening strategy in ENU mutagenesis is phenotype-driven, strenuous efforts are required to find the mutated genes. The identification and characterization of the Fitness 1 mutant mice can be a good example. Thirteen years ago, 5 lines of mutant mice (showing a fitness/runting syndrome, defined as fitness-1 (fit-1) mutants) were identified a through regional, recessive screen (Rinchik et al., 1990). Genomic locus was narrowed down to approximately the $530 \mathrm{~kb}$ sub-region between the eed and exed loci on chromosome 7 (Potter et al., 1995). Later, it was shown that these mutations in fit-1 locus resulted in defective hematopoiesis (Potter et al., 1997). This year it was finally identified that the mutated gene was a phosphatidylinositol-binding clathrin assembly protein (PICALM) gene, whose product is involved in clathrinmediated endocytosis (Klebig et al., 2003).

Genotype-driven screening Although it is considerably difficult to find the mutated genes, genotype-based screening procedures were recently being employed using the highthroughput system. This strategy makes it possible to screen for mutations on a specific gene with the genomic DNAs from mutagenized mice with ENU (Coghill et al., 2002). Sperms, achieved from mutagenized mice with ENU in parallel, allow the rapid expansion of the mutant mice.

Conventional germ-cell mutagenesis with N-ethyl-Nnitrosourea (ENU) has some disadvantages, including the inability to monitor the mutation rate, and strain and locusspecific variations, as well as laborious mouse husbandry. ES cell-based mutagenesis procedures provide another shortcut (Chen et al., 2000; Munroe et al., 2000). After mutagenizing ES cells with ethylmethanesulphonate (EMS) or ENU, the RT-PCR products of HPRT were sequenced and the mutations at the HPRT locus were functionally assessed using 6thioguanine (6-TG). The results from these studies indicated 


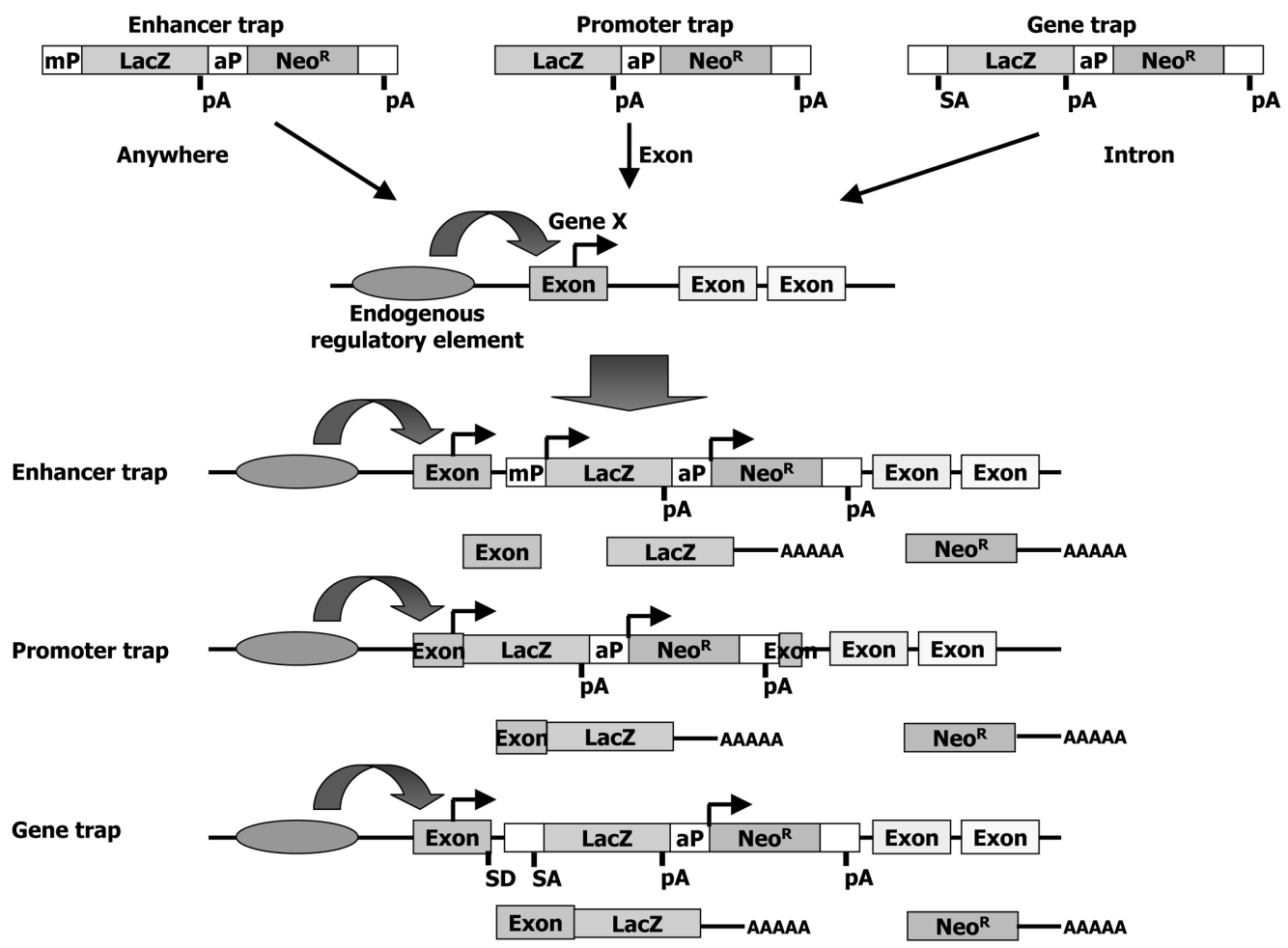

Fig. 3. Basic trap vectors and principles. Enhancer trap vector. Due to the nature of the enhancer trap vectors, it seems that the lossof-function mutation is not efficiently produced by the enhancer trap. For this reason, it has not been widely used in mice. Promoter trap vector. It must be integrated into the exonic sequence downstream of an endogenous promoter, which drives the reporter gene to be transcribed as a hybrid fused to the exonic sequence of the integration site. Gene trap vector. It has an additional splicing acceptor site upstream of the reporter gene-coding region. In contrast to the promoter trap, the gene trap is aimed at introns. Due to this splicing acceptor site, the upstream exonic sequence of the integration site and reporter gene sequence are fused by RNA splicing. mP, minimal promoter; ap, autonomous promoter such as pgk or beta actin promoter.

that this approach was plausible and could be used to generate mutant mice of various alleles from these ES cells. Using this strategy, the mutations on non-selectable genes, such as Smad2 and Smad4, were identified through high-throughput genotype-based screening (Vivian et al., 2002).

In conclusion, these approaches can be used to rapidly identify the allelic series of ENU-induced mutations on a specific gene, thus indicating that ENU-mediated mutagenesis can be applied to both the genotype-driven and phenotypedriven mutant screening in the post-genomic era.

\section{Gene Trap Mutagenesis}

Gene trapping is used to introduce a certain type of insertional mutation in ES cells, which is finally incorporated into the mouse genome (Fig. 3). Thus, ES cells provide the route through which the trapped genetic mutations can be induced in mouse genome (Stanford et al., 2001). Since the inserted gene-trap vectors can be used as a probe, the major screening strategy is genotype-driven. The gene-trap vectors have been modified in various ways to increase the trapping efficiency and identify the mutations in a specific group of genes. In this section, we will discuss some of the examples of its modifications and applications.

Polyadenylation trapping (polyA trapping) Since the promoterless reporter genes are used in both promoter- and gene-trap vectors, we can isolate only the genes that are transcriptionally active in undifferentiated ES cells. On the other hand, Polyadenylation trapping (polyA trapping) can isolate the genes which are not expressed in undifferentiated ES cells (Yoshida et al., 1995). The PolyA-trap vector has no polyadenylation signal. Instead, it has a splicing donor signal at the end of the reporter gene. Splicing between this donor and its acceptor on the downstream exon of the integrated site could produce a hybrid reporter transcript fused to the 3' region containing the polyadenylation signal. Even without the expression of the reporter gene, the 3 ' region that fused to the gene has enabled researchers to identify the mutated gene easily via 3' Rapid Amplification of cDNA End (RACE) in undifferentiated ES cells.

Expression trap As already mentioned, ES cells are pluripotent. Another strategy makes use of this character of ES cells for isolation of the trapped genes that are involved in 
specific developmental stages or expressed in the specialized organ or cell lineages (Stanford et al., 1998). By assaying the expression of reporter genes in differentiated ES cells, researchers could trap genes that are transcriptionally active in a specific cell lineage.

Secretory trap In order to trap some specialized gene groups, modified gene trap strategies have been applied. For example, using a "secretory trap", it is possible to identify the genes encoding secreted and membrane-spanning proteins (Skarnes et al., 1995). A secretory trap is accomplished by capturing the N-terminal signal sequence of an endogenous gene to generate an active beta-geo fusion protein in cytosol. This secretory trap strategy has been successfully conducted in mice (Mitchell et al., 2001).

Gene trap for distinguishing transcriptionally-responsive genes from constitutively-expressing genes The use of nitroreductase-fused EGFP as a reporter affords scientists to trap genes, transcriptionally responsive (Medico et al., 2001). The EGFP moiety enabled them to select only the ES cell clones into which gene trap vectors were integrated. The undifferentiated ES cells actively expressing EGFP were then screened using FACS. Since nitroreductase moiety, when expressed, acts as a negative selection marker in the presence of metronidazol by killing cells, then transcriptionallyresponsive genes could be distinguished from constitutivelyexpressing genes.

Though a gene trap vector randomly induces insertional mutations, there exists both vector-independent and vectorspecific hot spots for insertion. Since it was recently observed that more than $50 \%$ of these hot spots were vector-specific, a combination of gene-trap vectors must be used in order to saturate the mouse genome with gene-trap insertions (Hansen et al., 2003).

\section{Conclusions and Perspectives}

The development of mutagenesis strategies and the organic or systematical applications of these tools will pave the way for the functional analysis of each gene in vivo. Random mutagenesis strategies, such as ENU- and gene trapmutagenesis, have been developed to increase the number of mutant mice. By applying ENU mutagenesis on mice or ES cells, a series of phenotypically-interesting mutant alleles could be obtained without any assumption on the identity of a gene. The gene-trap with its high mutation frequencies allowed scientists to identify the mutated genes with relative ease. Now, the researchers could easily obtain mutant mice from stocks of the large-scale mutagenesis programs employing these types of random mutation, thereby accelerating the functional studies of the mouse genome.

However, the mutations from these strategies are practically limited, and so-called designer mice are needed to achieve the precise in vivo characterization of the various functional aspects of a gene. In this respect, transgenic technology is considered as an invaluable tool to conduct the studies related to the functional genomics in mice. A DNA fragment, microinjected into pronuclei, can reprogram the genome, which induces the transformation of the mouse through the transmission of a new mutation. By observing the phenotypes of transgenic mice, the in vivo effects of each mutation can be deduced, which would eventually help scientists to refine the functions of the gene. With the combination of various techniques such as RNAi, transgenic technology is beginning to widen its application range.

Even with all the versatilities of the strategies previously described, only gene targeting could definitively change the genetic status without any leakiness. As is already being done in other organisms such as yeast and C. elegans, it is expected that each gene in the mouse genome will be inevitably targeted in the future for its functional analysis in vivo.

Acknowledgments This work was supported by grants from the 21C Frontier Functional Human Genome Project from MOST, NRL (M1-0203-00-0108), KOSEF (SRCMTRC), and Samsung Biomedical Research Institute.

\section{References}

Adams, J. M., Harris, A. W., Pinkert, C. A., Corcoran, L. M., Alexander, W. S., Cory, S., Palmiter, R. D. and Brinster, R. L. (1985) The c-myc oncogene driven by immunoglobulin enhancers induces lymphoid malignancy in transgenic mice. Nature 318, 533-538.

Allsopp, R. C., Morin, G. B., Horner, J. W., DePinho, R., Harley, C. B. and Weissman, I. L. (2003) Effect of TERT overexpression on the long-term transplantation capacity of hematopoietic stem cells. Nat. Med. 9, 369-371.

Antonson, P., Schuster, G. U., Wang, L., Rozell, B., Holter, E., Flodby, P., Treuter, E., Holmgren, L. and Gustafsson, J. A. (2003) Inactivation of the nuclear receptor coactivator RAP250 in mice results in placental vascular dysfunction. Mol. Cell Biol. 23, 1260-1268.

Artandi, S. E., Alson, S., Tietze, M. K., Sharpless, N. E., Ye, S., Greenberg, R. A., Castrillon, D. H., Horner, J. W., Weiler, S. R., Carrasco, R. D. and DePinho, R. A. (2002) Constitutive telomerase expression promotes mammary carcinomas in aging mice. Proc. Natl. Acad. Sci. USA 99, 8191-8196.

Balling, R. (2001) ENU Mutagenesis: Analyzing Gene Function in Mice. Annu. Rev. Genomics Hum. Genet. 2, 463-492.

Barton, G. M. and Medzhitov, R. (2002) Retroviral delivery of small interfering RNA into primary cells. Proc. Natl. Acad. Sci. USA 99, 14943-14945.

Brinster, R. L., Chen, H. Y., Messing, A., van Dyke, T., Levine, A. J. and Palmiter, R. D. (1984) Transgenic mice harboring SV40 T-antigen genes develop characteristic brain tumors. Cell 37, 367-379.

Brummelkamp, T. R., Bernards, R. and Agami, R. (2002) A system for stable expression of short interfering RNAs in mammalian cells. Science 296, 550-553. 
Cao, Y., Bonizzi, G., Seagroves, T. N., Greten, F. R., Johnson, R., Schmidt, E. V. and Karin, M. (2001) IKK $\alpha$ provides an essential link between RANK signaling and cyclin D1 expression during mammary gland development. Cell 107, 763775.

Caplen, N. J., Parrish, S., Imani, F., Fire, A. and Morgan, R. A. (2001) Specific inhibition of gene expression by small doublestranded RNAs in invertebrate and vertebrate systems. Proc. Natl. Acad. Sci. USA 98, 9742-9747.

Chen, Y., Yee, D., Dains, K., Chatterjee, A., Cavalcoli, J., Schneider, E., Om, J., Woychik, R. P. and Magnuson, T. (2000) Genotype-based screen for ENU-induced mutations in mouse embryonic stem cells. Nat. Genet. 24, 314-317.

Choi, Y. S., Hong, S. B., Jeon, H. K., Kim, E. J., Oh, W. J., Joe, S. Y., Han, J. S., Lee, M. J. and Lee, H. W. (2003) Insertional mutation in the intron 1 of Unc5h3 gene induces ataxic, lean and hyperactive phenotype in mice. Exp. Anim. 52, 273-283.

Coghill, E. L., Hugill, A., Parkinson, N., Davison, C., Glenister, P., Clements, S., Hunter, J., Cox, R. D. and Brown, S. D. (2002) A gene-driven approach to the identification of ENU mutants in the mouse. Nat. Genet. 30, 255-256.

Devroe, E. and Silver, P. A. (2002) Retrovirus-delivered siRNA. BMC Biotechnol. 2, 15.

Doetschman, T., Gregg, R. G., Maeda, N., Hooper, M. L., Melton, D. W., Thompson, S. and Smithies, O. (1987) Targeted correction of a mutant hprt gene in mouse embryonic stem cells. Nature 330, 576-578.

Dymecki, S. M. (1996) FLP recombinase promotes site-specific DNA recombination in embryonic stem cells and transgenic mice. Proc. Natl. Acad. Sci. USA 93, 6191-6196.

Efrat, S., Fusco-DeMane, D., Lemberg, H., Emran, O. A. and Wang, X. (1995) Conditional transformation of a pancreatic $\beta$ cell line derived from transgenic mice expressing a tetracyclineregulated oncogene. Proc. Natl. Acad. Sci. USA 92, 3576-3580.

Elbashir, S. M., Harborth, J., Lendeckel, W., Yalcin, A., Weber, K. and Tuschl, T. (2001) Duplexes of 21-nucleotide RNAs mediate RNA interference in cultured mammalian cells. Nature 411, 494-498.

Evans, M. J. and Kaufman, M. H. (1981) Establishment in culture of pluripotential cells from mouse embryos. Nature 292, 154156.

Fire, A., Xu, S., Montgomery, M. K., Kostas, S. A., Driver, S. E. and Mello, C. C. (1998) Potent and specific genetic interference by double-stranded RNA in Caenorhabditis elegans. Nature 391, 806-811.

Geng, Y., Whoriskey, W., Park, M. Y., Bronson, R. T., Medema, R. H., Li, T., Weinberg, R. A. and Sicinski, P. (1999) Rescue of cyclin D1 deficiency by knockin cyclin E. Cell 97, 767-777.

Gonzalez-Suarez, E., Samper, E., Ramirez, A., Flores, J. M., Martin-Caballero, J., Jorcano, J. L. and Blasco, M. A. (2001) Increased epidermal tumors and increased skin wound healing in transgenic mice overexpressing the catalytic subunit of telomerase, mTERT, in basal keratinocytes. EMBO J. 20, 26192630.

Gu, H., Marth, J. D., Orban, P. C., Mossmann, H. and Rajewsky, K. (1994) Deletion of a DNA polymerase $\beta$ gene segment in T cells using cell type-specific gene targeting. Science $\mathbf{2 6 5}, 103-$ 106.

Hammer, R. E., Swift, G. H., Ornitz, D. M., Quaife, C. J., Palmiter, R. D., Brinster, R. L. and MacDonald, R. J. (1987)
The rat elastase I regulatory element is an enhancer that directs correct cell specificity and developmental onset of expression in transgenic mice. Mol. Cell Biol. 7, 2956-2967.

Hanahan, D. (1985) Heritable formation of pancreatic beta-cell tumors in transgenic mice expressing recombinant insulin/ simian virus 40 oncogenes. Nature 315, 115-122.

Hannon, G. J. (2002) RNA interference. Nature 418, 244-251.

Hansen, J., Floss, T., van Sloun, P., Fuchtbauer, E. M., Vauti, F., Arnold, H. H., Schnutgen, F., Wurst, W., von Melchner, H. and Ruiz, P. (2003) A large-scale, gene-driven mutagenesis approach for the functional analysis of the mouse genome. Proc. Natl. Acad. Sci. USA 100, 9918-9922.

Hasuwa, H., Kaseda, K., Einarsdottir, T. and Okabe, M. (2002) Small interfering RNA and gene silencing in transgenic mice and rats. FEBS Lett. 532, 227-230.

Kile, B. T., Hentges, K. E., Clark, A. T., Nakamura, H., Salinger, A. P., Liu, B., Box, N., Stockton, D. W., Johnson, R. L., Behringer, R. R., Bradley, A. and Justice, M. J. (2003) Functional genetic analysis of mouse chromosome 11. Nature 425, 81-86.

Kim, S. W., Cheong, C., Sohn, Y. C., Goo, Y. H., Oh, W. J., Park, J. H., Joe, S. Y., Kang, H. S., Kim, D. K., Kee, C., Lee, J. W. and Lee, H. W. (2002) Multiple developmental defects derived from impaired recruitment of ASC-2 to nuclear receptors in mice: implication for posterior lenticonus with cataract. $\mathrm{Mol}$. Cell Biol. 22, 8409-8414.

Klebig, M. L., Wall, M. D., Potter, M. D., Rowe, E. L., Carpenter, D. A. and Rinchik, E. M. (2003) Mutations in the clathrinassembly gene Picalm are responsible for the hematopoietic and iron metabolism abnormalities in fit1 mice. Proc. Natl. Acad. Sci. USA 100, 8360-8365.

Kuang, S. Q., Liao, L., Zhang, H., Pereira, F. A., Yuan, Y., DeMayo, F. J., Ko, L. and Xu, J. (2002) Deletion of the cancer-amplified coactivator AIB3 results in defective placentation and embryonic lethality. J. Biol. Chem. 277, 45356-45360.

Kunath, T., Gish, G., Lickert, H., Jones, N., Pawson, T. and Rossant, J. (2003) Transgenic RNA interference in ES cellderived embryos recapitulates a genetic null phenotype. Nature Biotechnol. 21, 559-561.

Kuro-o, M., Matsumura, Y., Aizawa, H., Kawaguchi, H., Suga, T., Utsugi, T., Ohyama, Y., Kurabayashi, M., Kaname, T., Kume, E., Iwasaki, H., Iida, A., Shiraki-Iida, T., Nishikawa, S., Nagai, R. and Nabeshima, Y. I. (1997) Mutation of the mouse klotho gene leads to a syndrome resembling ageing. Nature 390, 4551.

Lakso, M., Sauer, B., Mosinger, B. Jr., Lee, E. J., Manning, R. W., Yu, S. H., Mulder, K. L. and Westphal, H. (1992) Targeted oncogene activation by site-specific recombination in transgenic mice. Proc. Natl. Acad. Sci. USA 89, 6232-6236.

Lewandoski, M. (2001) Conditional control of gene expression in the mouse. Nat. Rev. Genet. 2, 743-755.

Mansour, S. L., Thomas, K. R. and Capecchi, M. R. (1988) Disruption of the protooncogene int-2 in mouse embryo-derived stem cells: A general strategy for targeting mutations to nonselectable genes. Nature 336, 348-352.

Matsukura, S., Jones, P. A. and Takai, D. (2003) Establishment of conditional vectors for hairpin siRNA knockdowns. Nucleic Acids Res. 31, e77.

Medico, E., Gambarotta, G., Gentile, A., Comoglio, P. M. and 
Soriano, P. (2001) A gene trap vector system for identifying transcriptionally responsive genes. Nat. Biotechnol. 19, 579582.

Mitchell, K. J., Pinson, K. I., Kelly, O. G., Brennan, J., Zupicich, J., Scherz, P., Leighton, P. A., Goodrich, L. V., Lu, X., Avery, B. J., Tate, P., Dill, K., Pangilinan, E., Wakenight, P., TessierLavigne, M. and Skarnes, W. C. (2001) Functional analysis of secreted and transmembrane proteins critical to mouse development. Nat. Genet. 28, 241-249.

Mousses, S., Caplen, N. J., Cornelison, R., Weaver, D., Basik, M., Hautaniemi, S., Elkahloun, A. G., Lotufo, R. A., Choudary, A., Dougherty, E. R., Suh, E. and Kallioniemi, O. (2003) RNAi microarray analysis in cultured mammalian cells. Genome Res. 13, 2341-2347.

Muller, U. (1999) Ten years of gene targeting: targeted mouse mutants, from vector design to phenotype analysis. Mech. Dev. 82, 3-21.

Munroe, R. J., Bergstrom, R. A., Zheng, Q. Y., Libby, B., Smith, R., John, S. W., Schimenti, K. J., Browning, V. L. and Schimenti, J. C. (2000) Mouse mutants from chemically mutagenized embryonic stem cells. Nat. Genet. 24, 318-321.

Nagy, A., Rossant, J., Nagy, R., Abramow-Newerly, W. and Roder, J. C. (1993) Derivation of completely cell culture-derived mice from early passage embryonic stem cells. Proc. Natl. Acad. Sci. USA 90, 8424-8428.

Oh, H., Taffet, G. E., Youker, K. A., Entman, M. L., Overbeek, P. A., Michael, L. H. and Schneider, M. D. (2001) Telomerase reverse transcriptase promotes cardiac muscle cell proliferation, hypertrophy, and survival. Proc. Natl. Acad. Sci. USA 98, 10308-10313.

Orban, P. C., Chui, D. and Marth, J. D. (1992) Tissue- and sitespecific DNA recombination in transgenic mice. Proc. Natl. Acad. Sci. USA 89, 6861-6865.

Ornitz, D. M., Palmiter, R. D., Hammer, R. E., Brinster, R. L., Swift, G. H. and MacDonald, R. J. (1985) Specific expression of an elastase-human growth hormone fusion gene in pancreatic acinar cells of transgenic mice. Nature 313, 600-602.

Paddison, P. J., Caudy, A. A., Bernstein, E., Hannon, G. J. and Conklin, D. S. (2002) Short hairpin RNAs (shRNAs) induce sequence-specific silencing in mammalian cells. Genes Dev. 16, 948-958.

Palmiter, R. D., Brinster, R. L., Hammer, R. E., Trumbauer, M. E., Rosenfeld, M. G., Birnberg, N. C. and Evans, R. M. (1982) Dramatic growth of mice that develop from eggs microinjected with metallothionein-growth hormone fusion genes. Nature $\mathbf{3 0 0}$, 611-615.

Palmiter, R. D., Behringer, R. R., Quaife, C. J., Maxwell, F., Maxwell, I. H. and Brinster, R. L. (1987) Cell lineage ablation in transgenic mice by cell-specific expression of a toxin gene. Cell 50, 435-443.

Potter, M. D., Klebig, M. L., Carpenter, D. A. and Rinchik, E. M. (1995) Genetic and physical mapping of the fitness 1 (fit1) locus within the Fes-Hbb region of mouse chromosome 7. Mamm. Genome 6, 70-75.

Potter, M. D., Shinpock, S. G., Popp, R. A., Godfrey, V., Carpenter, D. A., Bernstein, A., Johnson, D. K. and Rinchik, E. M. (1997) Mutations in the murine fitness 1 gene result in defective hematopoiesis. Blood 90, 1850-1857.

Rinchik, E. (2000) Developing genetic reagents to facilitate recovery, analysis, and maintenance of mouse mutations.
Mamm. Genome 11, 489-499.

Rinchik, E. M., Carpenter, D. A. and Selby, P. B. (1990) A strategy for fine-structure functional analysis of a 6- to 11centimorgan region of mouse chromosome 7 by high-efficiency mutagenesis. Proc. Natl. Acad. Sci. USA 87, 896-900.

Robertson, E., Bradley, A., Kuehn, M. and Evans, M. (1986) Germ-line transmission of genes introduced into cultured pluripotential cells by retroviral vector. Nature 323, 445-448.

Rubinson, D. A., Dillon, C. P., Kwiatkowski, A. V., Sievers, C., Yang, L., Kopinja, J., Rooney, D. L., Ihrig, M. M., McManus, M. T., Gertler, F. B., Scott, M. L. and Van Parijs, L. (2003) A lentivirus-based system to functionally silence genes in primary mammalian cells, stem cells and transgenic mice by RNA interference. Nat. Genet. 33, 401-406.

Saito, M., Iwawaki, T., Taya, C., Yonekawa, H., Noda, M., Inui, Y., Mekada, E., Kimata, Y., Tsuru, A. and Kohno, K. (2001) Diphtheria toxin receptor-mediated conditional and targeted cell ablation in transgenic mice. Nat. Biotechnol. 19, 746-750.

Shinagawa, T. and Ishii, S. (2003) Generation of Ski-knockdown mice by expressing a long double-strand RNA from an RNA polymerase II promoter. Genes Dev. 17, 1340-1345.

Skarnes, W. C., Moss, J. E., Hurtley, S. M. and Beddington, R. S. (1995) Capturing genes encoding membrane and secreted proteins important for mouse development. Proc. Natl. Acad. Sci. USA 92, 6592-6596.

Stacey, A., Bateman, J., Choi, T., Mascara, T., Cole, W. and Jaenisch, R. (1988) Perinatal lethal osteogenesis imperfecta in transgenic mice bearing an engineered mutant pro-alpha 1(I) collagen gene. Nature 332, 131-136.

Stanford, W. L., Caruana, G., Vallis, K. A., Inamdar, M., Hidaka, M., Bautch, V. L. and Bernstein, A. (1998) Expression trapping: identification of novel genes expressed in hematopoietic and endothelial lineages by gene trapping in ES cells. Blood 92, 4622-4631.

Stanford, W. L., Cohn, J. B. and Cordes, S. P. (2001) Gene-trap mutagenesis: past, present and beyond. Nat. Rev. Genet. 2, 756768.

Thomas, K. R. and Capecchi, M. R. (1987) Site-directed mutagenesis by gene targeting in mouse embryo-derived stem cells. Cell 51, 503-512.

Thomas, J. W., Lamantia, C. and Magnuson, T. (1998) X-rayinduced mutations in mouse embryonic stem cells. Proc. Natl. Acad. Sci. USA 95, 1114-1119.

Tiscornia, G., Singer, O., Ikawa, M. and Verma, I. M. (2003) A general method for gene knockdown in mice by using lentiviral vectors expressing small interfering RNA. Proc. Natl. Acad. Sci. USA 100, 1844-1848.

Vivian, J. L., Chen, Y., Yee, D., Schneider, E. and Magnuson, T. (2002) An allelic series of mutations in Smad2 and Smad4 identified in a genotype-based screen of N-ethyl-Nnitrosourea-mutagenized mouse embryonic stem cells. Proc. Natl. Acad. Sci. USA 99, 15542-15547.

Vooijs, M., van der Valk, M., te Riele, H. and Berns, A. (1998) Flp-mediated tissue-specific inactivation of the retinoblastoma tumor suppressor gene in the mouse. Oncogene 17, 1-12.

Wiznerowicz, M. and Trono, D. (2003) Conditional suppression of cellular genes: lentivirus vector-mediated drug-inducible RNA interference. J. Virol. 77, 8957-8961.

Yoshida, M., Yagi, T., Furuta, Y., Takayanagi, K., Kominami, R., Takeda, N., Tokunaga, T., Chiba, J., Ikawa, Y. and Aizawa, S. 
(1995) A new strategy of gene trapping in ES cells using 3'RACE. Transgenic Res. 4, 277-287.

Yu, Y. and Bradley, A. (2001) Engineering chromosomal rearrangements in mice. Nat. Rev. Genet. 2, 780-790.
Zheng, B., Sage, M., Cai, W. -W., Thompson, D. M., Tavsanli, B. C., Cheah, Y. -C. and Bradley, A. (1999) Engineering a mouse balancer chromosome. Nat. Genet. 22, 375-378. 